\title{
Predictions and Magnetic Characterization of M-type Hexaferrites Doped with Two Cations in the $\mathrm{Fe}^{3+}$ Site
}

\author{
Pedro Antonio Mariño-Castellanos ${ }^{1, ~ *, ~ V a l e r y ~ A l e x e v i c h ~ L a p s h i n s k y ~}{ }^{2}$, Carlos Pupo ${ }^{1,2}$, \\ Arles Vega-García ${ }^{1}$, Eduardo Fernández-Cruz ${ }^{3}$, Braddy Ivan Jimenez-Morales ${ }^{4}$ \\ ${ }^{1}$ University of Holguin, Holguin, Cuba \\ ${ }^{2}$ Peoples Friendship University of Russia, Moscow, Russian Federation \\ ${ }^{3}$ Department of Physics, University of Camagüey, Camagüey, Cuba \\ ${ }^{4}$ Physics Institute, Kazan Federal University, Kazan, Russian Federation
}

Email address:

pamarcast@uho.edu.cu (P. A. Mariño-Castellanos)

${ }^{*}$ Corresponding author

\section{To cite this article:}

Pedro Antonio Mariño-Castellanos, Valery Alexevich Lapshinsky, Carlos Pupo, Arles Vega-García, Eduardo Fernández-Cruz, Braddy Ivan Jimenez-Morales. Predictions and Magnetic Characterization of M-type Hexaferrites Doped with Two Cations in the Fe ${ }^{3+} \mathrm{Site}^{\text {American }}$ Journal of Engineering and Technology Management. Vol. 4, No. 6, 2019, pp. 83-90. doi: 10.11648/j.ajetm.20190406.12

Received: September 29, 2019; Accepted: November 5, 2019; Published: December 2, 2019

\begin{abstract}
A theoretical and experimental study of magnetic properties of M-type barium hexaferrites, doped with two cations in the $\mathrm{Fe}^{3+}$ sites: $\left(\mathrm{Co}^{3+}, \mathrm{Al}^{3+}\right),\left(\mathrm{Co}^{2+}, \mathrm{Ti}^{4+}\right)$ and $\left(\mathrm{Co}^{2+}, \mathrm{Sn}^{4+}\right)$ is reported. The theoretical predictions were made using the generalization of a model on the distribution of dopants in the crystallographic sites under study. This approach allows a better interpretation of the kinetics of obtaining the samples from the structural and magnetic characterization. In the reported model, the maximum limit refers to theoretical optimum parameters. With these concepts, were established quantitative relationships between dopant amount per crystallographic site and magnetic properties predicted of these systems. The comparison between the experimental values and the predicted magnitudes showed that, in general, the samples were obtained with parameters obtained close to the theoretical ones for traditional ceramic methods. Such an approach is rarely taken into account in the reported bibliography.
\end{abstract}

Keywords: Sol-Gel Methods, Magnetic Properties, Hexaferrite, Theoretical Predictions, Rietveld Refinement

\section{Introduction}

The magnetic structure of BaM is described by the Gorter model as an uniaxial collinear ferrite [1], with three spin-up (2a, 2b, 12k) and two spin-down (4fiv, 4fvi) sublattices along the c-axis. It is a ferromagnetic material with high coercitivity, magnetic permeability, saturation magnetization and magneto-crystalline anisotropy along the c-axis [2]. Its magnetic properties are functions of the crystalline microstructure $[2,3]$.

Several investigations have been carried out to study the influence of various dopants on its physical properties of Mtype hexaferrites. The microstructural modification of the barium hexaferrite structure, leads to change in magnetic, and microwave properties, an effective method is the doping of $\mathrm{Ba}^{2+}$ or $\mathrm{Fe}^{3+}$ cations sites with other metallic cations. During the past few years it becomes possible to synthesize some substitution M-type hexaferrites in which coercivity decline effectively with cations dopant. To achieve this objective, we have, the doping of transition metals, e.g., $\mathrm{Co}^{2+}, \mathrm{Mg}^{2+}, \mathrm{Zn}^{2+}$, $\mathrm{Ni}^{2+}, \mathrm{Al}^{3+}, \mathrm{Cr}^{3+}, \mathrm{Ti}^{4+}, \mathrm{Zr}^{4+}$, and $\mathrm{Sn}^{4+}$, which has been investigated by several researchers to modify different properties of M-type hexaferrite [4, 5].

However, only few theoretical studies about M-type hexaferrites have been reported regarding in relation with the cation distribution in the different crystallographic sites of this crystal structure $[4,6]$.

The objective of the present investigation is to establish the dependences on the microstructure of the magnetic properties of $\mathrm{M}$-type barium hexaferrites doped with $\mathrm{CO}^{3+}$, $\left.\mathrm{Al}^{3+}\right),\left(\mathrm{Co}^{2+}, \mathrm{Ti}^{4+}\right)$ and $\left(\mathrm{Co}^{2+}, \mathrm{Sn}^{4+}\right)$ in the iron sites and their comparison with the theoretical predictions obtained of the 
generalization of a model on the distribution of dopants in the crystallographic sites under study.

\section{Materials and Methods}

The samples were obtained by the traditional ceramic method, using $\mathrm{BaCO}_{3}, \mathrm{Fe}_{2} \mathrm{O}_{3}, \mathrm{Al}_{2} \mathrm{O}_{3}, \mathrm{TiO}_{2}, \mathrm{CoO}, \mathrm{Co}_{2} \mathrm{O}_{3}$ and $\mathrm{SnO}_{2}$ as starting materials. They were mixed in the required stoichiometric ratio according to the chemical formula $\mathrm{BaFe}_{(12-2 x \mathrm{Dj})} \mathrm{A}_{\mathrm{xDj}} \mathrm{B}_{\mathrm{xDj}} \mathrm{O}_{19}$ where $\mathrm{A}_{\mathrm{xDj}}=\mathrm{Al}^{3+}, \mathrm{Ti}^{4+}, \mathrm{Sn}^{4+}$ and $\mathrm{B}_{\mathrm{xDj}}=\mathrm{Co}^{3+}, \mathrm{Co}^{2+}$.

The structural characterization was carried out from the $\mathrm{X}$ rays diffraction patterns, and the wavelength of radiation was $\operatorname{Co}\left[\lambda\left(\mathrm{k}_{\alpha 1}\right)\right]=1.7889 \AA$.

The qualitative phase analysis was carried out by Hanawalt method and software Match Phase Identification from Powder Diffraction. Each diffraction pattern was refined by Rietveld method, using the Fullprof software [4, 6, 7].

The magnetic study were characterized by using of a rotating sample magnetometer (RSM) $[4,6,7]$.

\section{The Phenomenological Model}

$$
U_{\text {rsite }}=\frac{1214 \gamma Z_{C} z_{a}}{r_{C}+r_{a}}\left[\left(1-\frac{34.5}{r_{c}+r_{a}}\right)+0.00453\left(r_{C}+r_{a}\right)\right][\mathrm{KJ} / \mathrm{mol}]
$$

Where $U_{\text {rsite }}$ - reticular energy per site; $[\mathrm{r}]=10^{-12} \mathrm{~m} ; \gamma$ quantity of ions per formula unit; $Z_{c}$ - cation atomic number; $\mathrm{Z}_{\mathrm{a}}$ - anion atomic number; $\mathrm{rc}$ - cationic radius and ra anionic radius.

The dependence on dopant type (through Zc), quantity of dopant (through $\gamma$ ) and crystallographic sites (through rc and ra) are presented, theoretically demonstrating this dependence during the formation process of doped $\operatorname{BaM}[4,9]$.

Equation (4) allows theoretical calculation of the involved formation energy in the sites where the dopants and the cations number are distributed, using the expressions (1), (2)
The total quantity of dopants in each site $[4,6]$ is expressed by the previous model in the following way:

$$
C_{x D p, x D j}(i)=N(i)-C_{C}(x D p, x D j, i)
$$

and the quantity of each dopant per site:

$$
\begin{aligned}
C_{x D p}(x D j, i) & =C_{x D p, x D j}(i) P S_{x D p}(x D j, i) \\
C_{x D j}(x D p, i) & =C_{x D p, x D j}(i) P S_{x D j}(x D p, i)
\end{aligned}
$$

The expressions from (1) to (3) are similar to those used in [7] when data from Mössbauer spectrometry are interpreted for the case of doping with two dopants.

Until here, the results contributed by this model represent the values to obtain in the case of ideal substitutions with optimum obtaining parameters, which do not involve any obtaining method. However, when the dopant is included, the optimum obtaining parameters for un-doped hexaferrites are not the same that parameters for doped samples, and the magnitude of such variation depends on concentration and dopant type [4, 6], as it can be observed from the generalized Kapustinskii equation [8] for the energy, in function of each crystallographic site:

$$
\begin{gathered}
C_{C}(x D p, x D j, i)_{\min }=N(i)-\left[C_{x D p}(x D j, i)_{\max }+C_{x D j}(x D j, i)_{\max }\right] \\
C_{x D p}(x D j, i)_{\min }=C_{x D p}(x D j, i)_{\max } D R_{x D p}(x D j, i) \\
C_{x D j}(x D j, i)_{\min }=C_{x D j}(x D j, i)_{\max } D R_{x D j}(x D j, i) \\
C_{C}(x D p, x D j, i)_{\text {max }}=N(i)-\left[C_{x D p}(x D j, i)_{\min }+C_{x D j}(x D j, i)_{\min }\right]
\end{gathered}
$$

The equations from (5) to (10) allow the calculation of substitutes cations distribution for optimum obtaining parameters (maximum values) and when these parameters change as a function of temperature and calcination time (minimum values) [10]. Therefore, it is possible to predict the behavior of saturation magnetization MS, magnetocrystalline anisotropic constant $\mathrm{K} 1$ and coercitive field $\mathrm{HC}$.

Assuming a collinear arrangement of the magnetic domains in the Fe3+ sites, the theoretical value of MS [6, 9, 11] can be calculated through the expression:

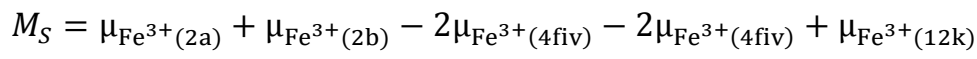

Where $\mu_{\mathrm{Fe}^{3+}}$ is the standard value of the $\mathrm{Fe}^{3+}$ magnetic moment, and since $\mu_{F e^{3+}}=5 \mu_{B}$ at $0 \mathrm{~K} \quad\left(\mu_{\mathrm{B}}\right.$-Bohr's magneton) will exist a total $\mathrm{MS}=20 \mu_{\mathrm{B}}$ per formula unit for

un-doped $\mathrm{BaFe}_{12} \mathrm{O}_{19}$ at this temperature. Extending the previous expression to the case of doped BaM with two dopants [4]:

$$
M_{S}=\sum_{i=1}^{5}\left(C C S_{i} \cdot \mu_{F e^{3+}}+C D S_{i} D_{j} \cdot \mu_{D j}+C D S_{i} D_{p} \cdot \mu_{D p}\right)
$$


For the axial-c anisotropy, $K_{1}>0$ and, usually, $K_{2}$ is negligible in comparison with $\mathrm{K}_{1}$. In this case, magnetocrystalline anisotropy field $\mathrm{H}_{\mathrm{an}}[4,12]$ is given by:

$$
H_{a n}=\frac{2 K_{1}}{M_{S}}
$$

and anisotropy constant $\mathrm{K}_{1}[4]$ is determined by:

$$
K_{1}=M_{\text {perp }}^{\max } H
$$

Where $M_{\text {perp }}^{\max }$ is the maximum perpendicular magnetization, measured in the RSM and $\mathrm{H}$ is the field under which the previous value was obtained.

According to the Stoner-Wohlfarth theory [13], coercivity is given by

$$
H_{C}=\alpha\left[\frac{2 K_{1}}{M_{S}}-D M_{S}\right]
$$

Where $\alpha$ is a geometrical parameter ( $\alpha=0.48$ in case of randomly oriented particles) and $\mathrm{D}$ is the demagnetization factor.

\section{Results and Discussion}

In the figures from 1 to 5 it is shown a comparison between cation's distributions experimentally determined and the predictions by the cation's distribution model, in the different $\mathrm{Fe}^{3+}$ sites.
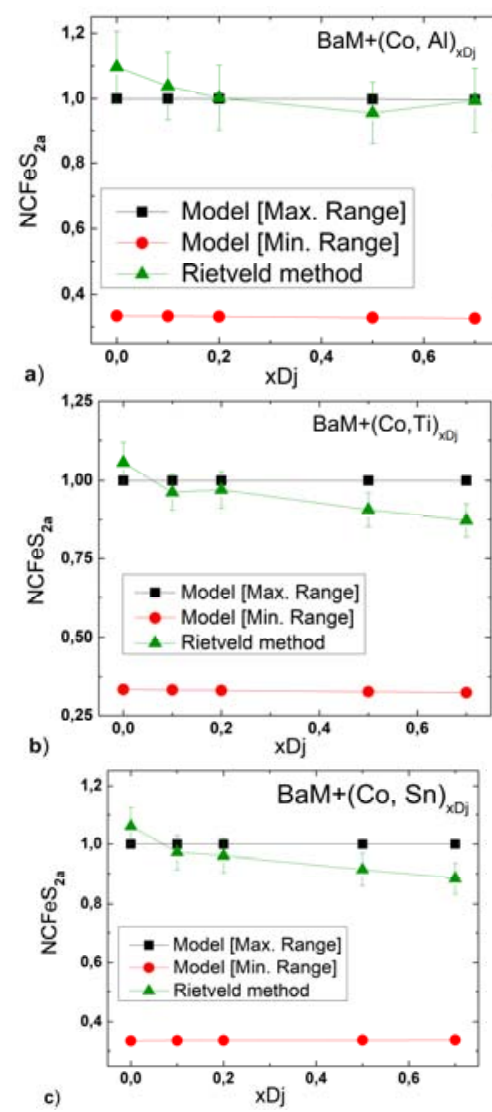

Figure 1. Comparison between the experimental results of the $\mathrm{Fe}^{3+}$ distribution determined by the Rietveld method and the predictions by the model for crystallographic site $2 a$, per formula unit.
In general, is observed a gradual decrease of the $\mathrm{Fe}^{3+}$ cation quantity in each site, whose dependence varies with the concentration and dopant type. The difference between maximum values of theoretical and experimental cation's distribution per site indicates that preparation parameters outdistance the optimum parameters. An abrupt change of this curve with $x \mathrm{Dj} \geq \mathrm{xDj}_{\text {critical }}$ is noticed.

The minimum predicted values refer the increase or decrease of required energy for compound formation.

The dopants occupy the spaces that were left by $\mathrm{Fe}^{3+}$ in each crystallographic position, in function of the preferential site for each one $[4,6]$.

In the figure 1 it is evident that in the doping range where doped BaM phase is in majority, this site is not a preferential one for the dopants considered in this investigation.

In the figure 2 is evident that for $\mathrm{BaM}+(\mathrm{Co}, \mathrm{Al})_{\mathrm{xDj}}$ the quantity of the ion $\mathrm{Fe} 3+$ decreases and it is not compensated by the dopant quantity that goes to this site.

For $\mathrm{BaM}+(\mathrm{Co}, \mathrm{Ti}) \times \mathrm{Xj}$ there is not variation for the iron ion until $\mathrm{xDj}$ is near the $\mathrm{xDj}_{\text {critical }}$. For $\mathrm{BaM}+(\mathrm{Co}, \mathrm{Sn})_{\mathrm{xDj}}$ the quantity of iron remains approximately constant in the whole range where this phase is in majority.

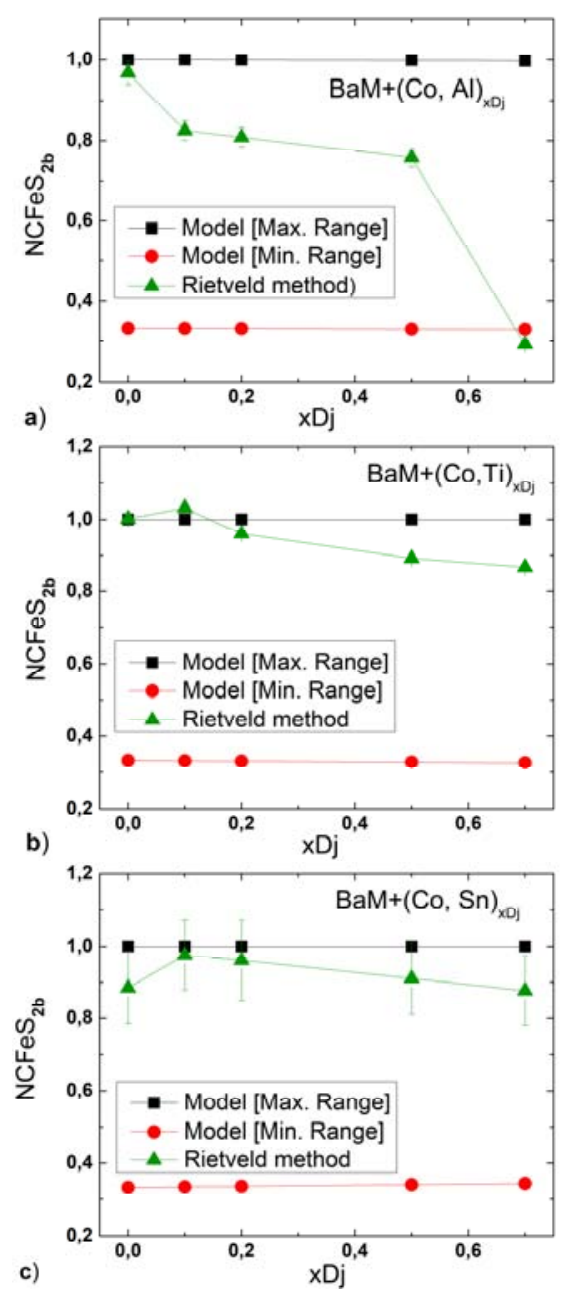

Figure 2. Comparison between the experimental results of the $\mathrm{Fe}^{3+}$ distribution determined by the Rietveld method and the predictions by the model for crystallographic site $2 b$, per formula unit. 
In the figure 3, it can be appreciated that the decrease of the iron cation quantity in the site 4fiv is appreciable, but small, only starting from xDjcritical for $\mathrm{BaM}+(\mathrm{Co}, \mathrm{Sn}) \mathrm{xDj}$.
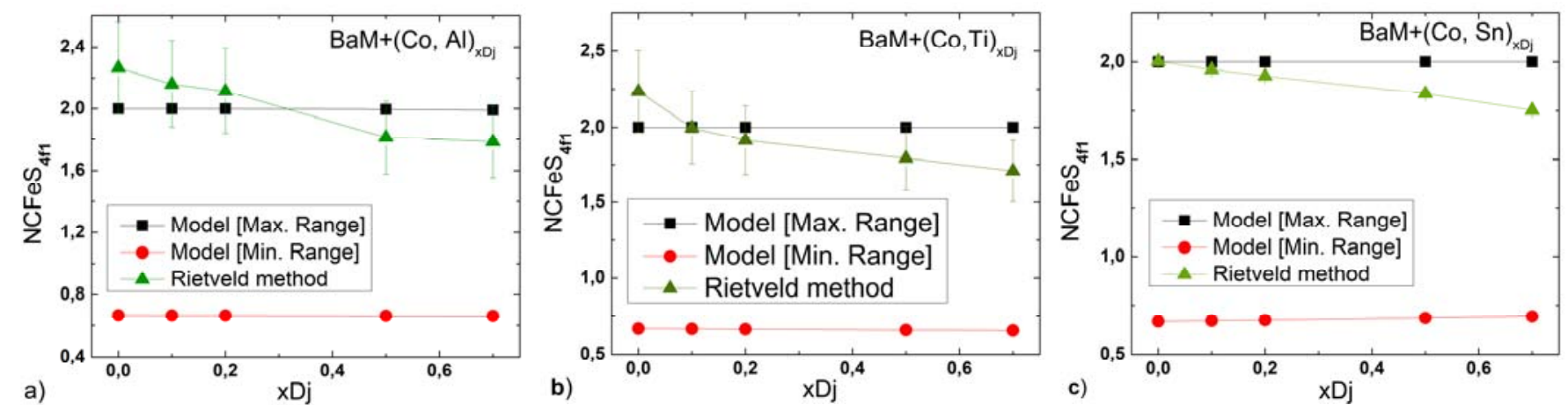

Figure 3. Comparison between the experimental results of the Fe ${ }^{3+}$ distribution determined by the Rietveld method and the predictions by the model for crystallographic site 4fiv, per formula unit.

The site 4 fvi has a similar behavior to the previous site 4 fiv (referred to figure 4 ), but now for $\mathrm{BaM}+(\mathrm{Co}, \mathrm{Ti})_{\mathrm{xDj}}$
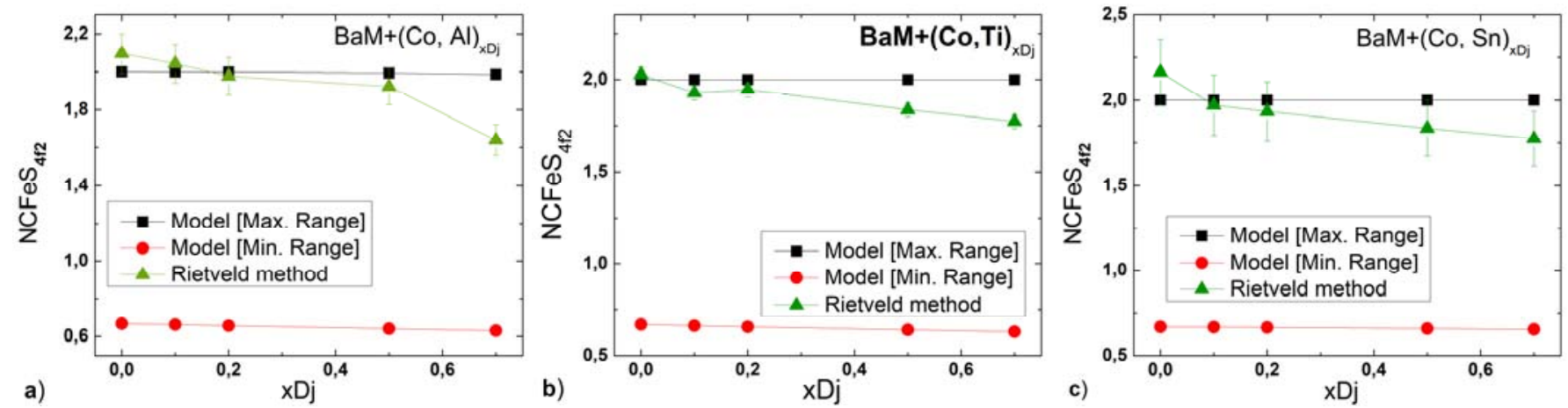

Figure 4. Comparison between the experimental results of the $\mathrm{Fe}^{3+}$ distribution determined by the Rietveld method and the predictions by the model for crystallographic site 4 fvi, per formula unit.

In the site $12 \mathrm{k}$ (referred to figure 5) the variations of the $\mathrm{Fe}^{3+}$ cations quantity begin to be important starting from $\mathrm{xDjcritical}$, although the behavior shows a monotonous decrease with the increment of the dopant quantity, being more evident for BaM $+(\mathrm{Co}, \mathrm{Sn})_{\mathrm{xDj}}$.
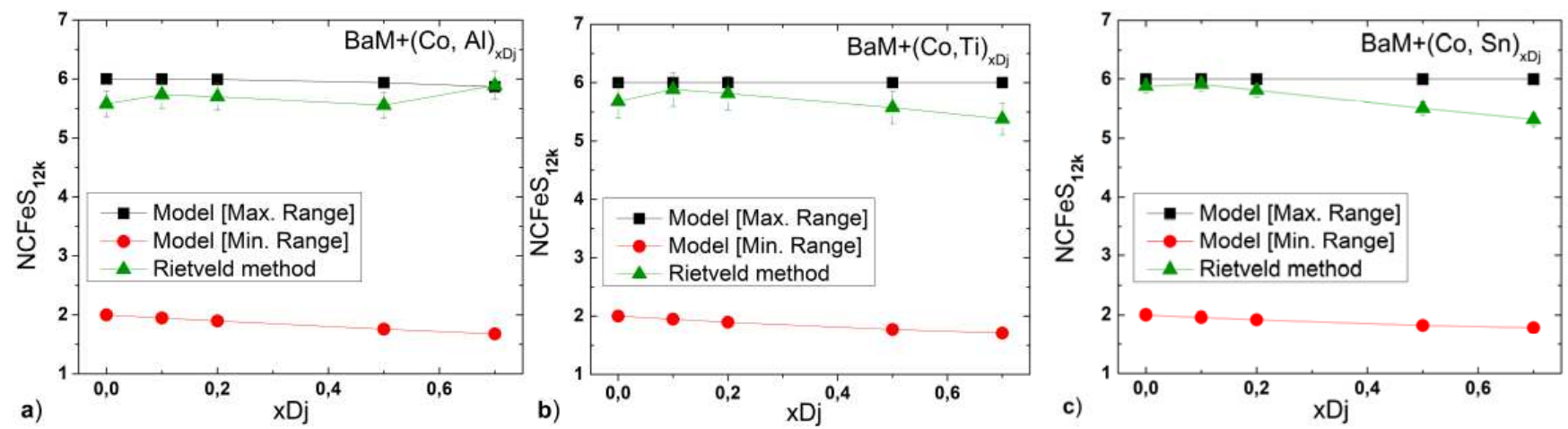

Figure 5. Comparison between the experimental results of the Fe ${ }^{3+}$ distribution determined by the Rietveld method and the predictions by the model for crystallographic site $12 k$, per formula unit.

In the figures 6 and 7 it is noticed that when the concentration is small, with the increment of the applied field the distance between a curve and another goes decreasing, and the point of maximum magnetic susceptibility is reached. 

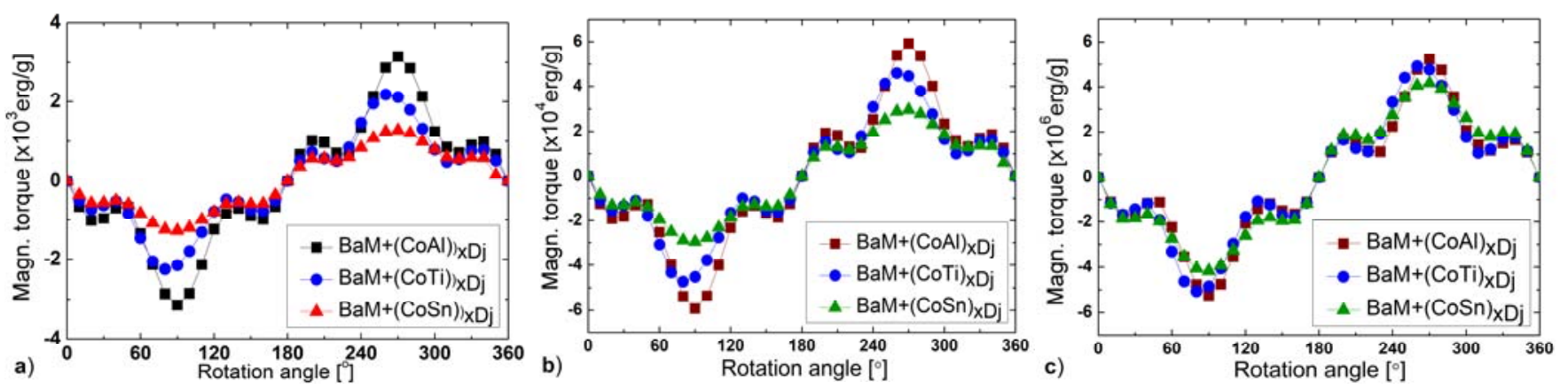

Figure 6. Magnetic torque in function of the rotation angle for the three systems, with $x D j=0.1$ and intensities of applied magnetic field of (a) 100 Oe, (b) 1000 Oe and (c) 9000 Oe.

A remarkable variation is observed in the magnetic torque magnitude when dopant concentration varies from 0.1 to 0.5 . This statement is demonstrated by the fact that for the applied field next to the maximum magnetic susceptibility, the three curves are similar, but they are different from one concentration level to another, due to other phases contribution for systems (Co, $\mathrm{Al})$ and (Co, Ti).

For $\mathrm{xDj}=0.5\left[\mathrm{xDj}=\mathrm{xDj}_{\text {critical }}\right.$ for systems $(\mathrm{Co}, \mathrm{Al})$ and $(\mathrm{Co}$, $\mathrm{Ti})$ ], it is observed that even at high intensity of applied field (9000Oe), the distance between the curves is still appreciable, due to the other phases contribution, morphology variations and crystallites distribution inside the matrix.
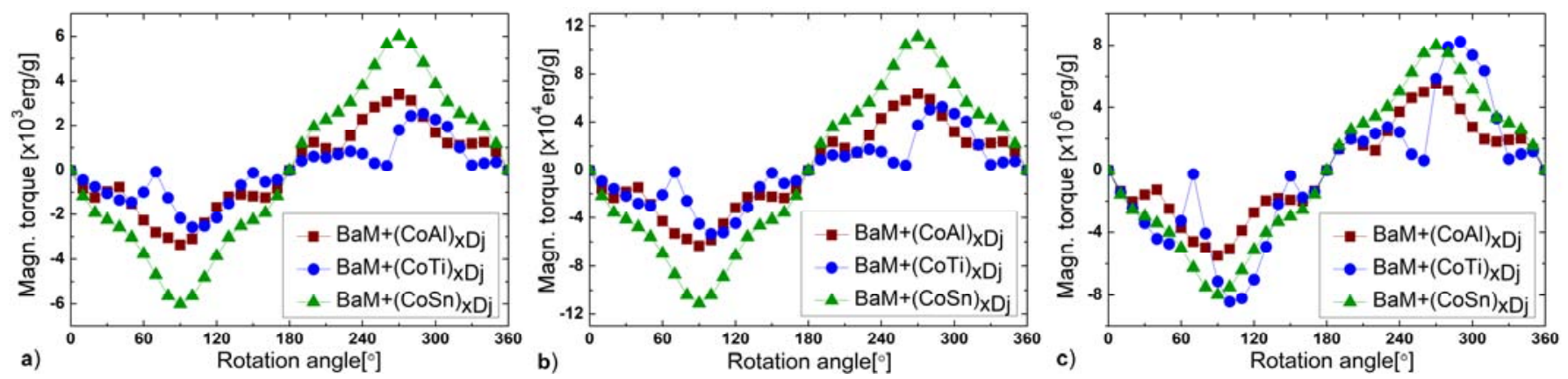

Figure 7. Magnetic torque in function of the rotation angle for the three systems, with $x D j=0.5$ and intensities of applied magnetic field of (a) 100 Oe, (b) 1000 Oe and (c) 9000 Oe.

In the figure 8 it is appreciated that theoretical prediction for the systems doped with (Co, $\mathrm{Al})$ and $(\mathrm{Co}, \mathrm{Ti})$ appropriately reflects the dependence of MS on the dopant quantity to $\mathrm{xDj}=\mathrm{xDj} \mathrm{j}_{\text {critical }}$. Starting from this value, doped $\mathrm{BaM}$ and cobalt monoferrite contributions are presented. The experimental values for these two systems are always smaller than the theoretically predicted values, since the dopant couples preferentially go to the position $12 \mathrm{k}$, and also as a result of the size and quantity of pores in these samples [4].
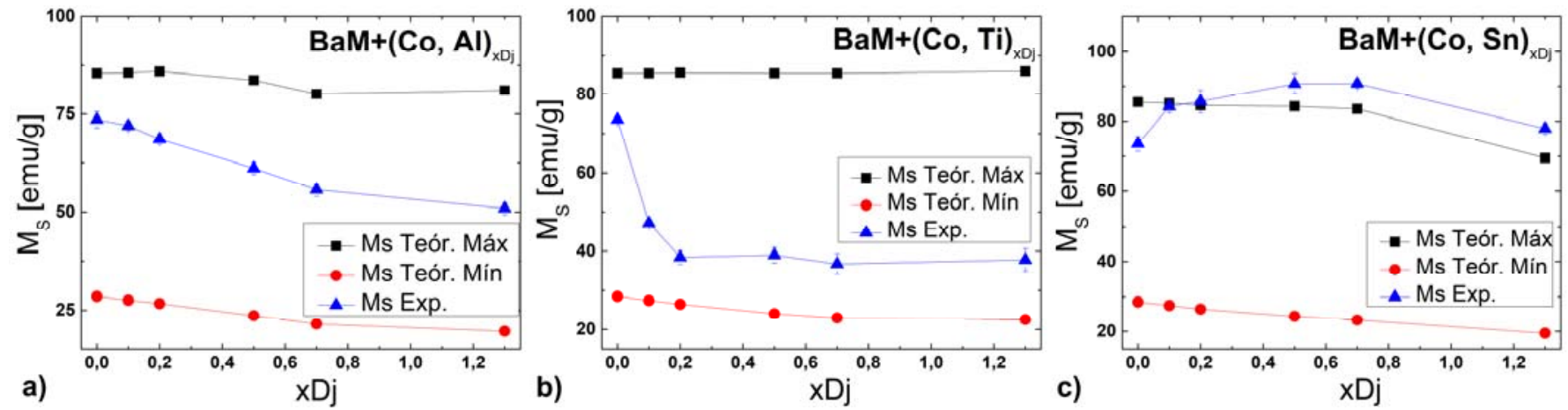

Figure 8. Experimental and theoretical saturation magnetization, in function of dopant amount per formula unit for the systems (a) BaM $+($ Co, Al)xDj, (b) $B a M+(C o, T i) x D j$ and (c) BaM + (Co, Sn) $x D j$.

In the figure 8 (c) it is remarkable that for the system $(\mathrm{Co}$, Sn), MS tends to increase with dopant concentration, differing from the theoretically predicted values. This is because starting from a concentration value $\mathrm{xDj}$ critical, the most probable process is not the formation of $\mathrm{BaM}$ doped with $(\mathrm{Co}, \mathrm{Sn})$ in the iron sites, but the formation of cobalt monoferrite, which is a soft magnet and it increases the magnetization for small values of $\mathrm{xDj}$ and tends to make constant the magnetization, starting from $x \mathrm{Djcritical}$. This value coincides with the value determined by XRD. 
MS depends on the contribution of each iron site to the total magnetization of the un-doped and doped samples with small dopant concentrations. Starting from values $\mathrm{xDj} \geq$ $\mathrm{xDj}_{\text {critical }}$ theoretical predictions will not be of the order of the experimental characterization, because these results depend on the formed phase's type.

In the figure 9 it is important to notice that although the

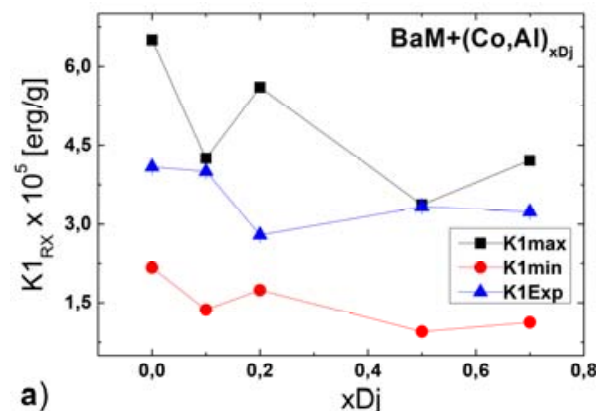

a)

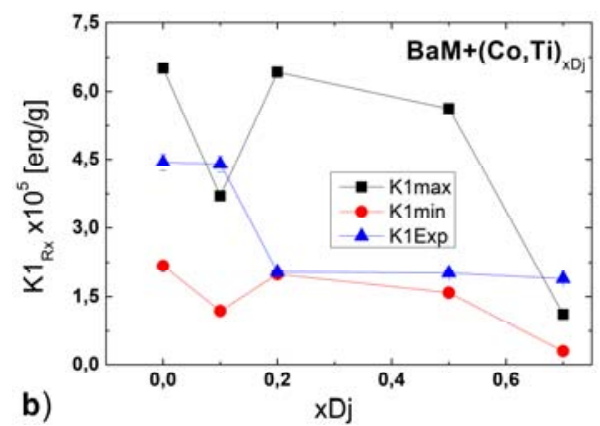

b)

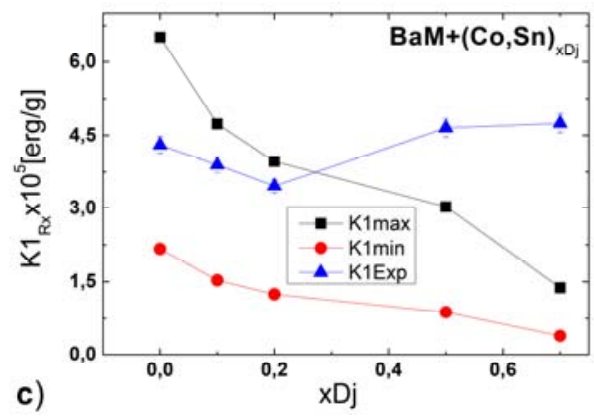

behavior of the theoretical and experimental densities is different, grain morphology and, consequently, the pores, only contribute to the anisotropy magnitude variation, not in the behavior. Therefore, we can infer that the dependence of this magnitude on the dopant amount is basically determined by dopant proportion in the sites $2 \mathrm{~b}$ and $12 \mathrm{k}$.
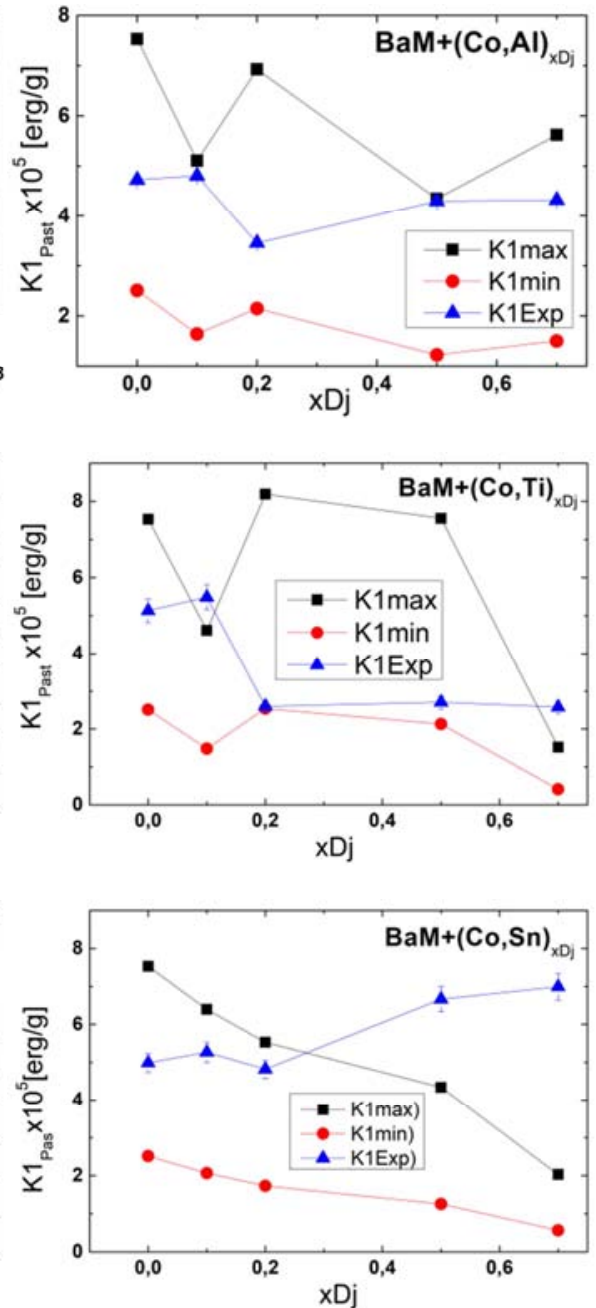

Figure 9. Comparison between theoretical and experimental anisotropy constant $K_{l}$, considering the BaM theoretical density and density of the pill, in function of the quantity of dopant per formula unit for the systems (a) BaM+(Co, Al) xDj, (b) BaM + (Co, Ti)xDj and (c) BaM + (Co, Sn)xDj.

For the systems $(\mathrm{Co}, \mathrm{Al})$ and $(\mathrm{Co}, \mathrm{Ti})$ there is an abrupt variation of the anisotropy with $0.1 \leq \mathrm{xDj} \leq 0.2$, since the systems change the anisotropy type from easy axis to easy plane, which is a fact expressed in an inverse way in the predictions due to the role of the anisotropy field in this dependence.

The strains in the cell have only a secondary influence in this behavior. A very strained crystallographic cell, an excessive size of pores and the cobalt monoferrite contribution are the physical causes that do not allow to separate the magneto-crystalline anisotropy and shape anisotropy that are presented in the compounds $\mathrm{BaM}+(\mathrm{Co}$, $\mathrm{Sn}) \mathrm{xDj}$.
The deviations between the theoretical predictions and the experimental results are given by three factors: 1) The presence of other phases, microinclusions and other surface defects that contribute to the extrinsic magnetic anisotropy, 2) The strains in the structure which start increasing from the value $\mathrm{xDj}_{\text {critical }}$ and 3) The pores inside the matrix, which tend to decrease the total magnetic anisotropy of the sample when they are big, and to increase this magnetic property when they are small.

In the figure 10 it is noticed that in all the systems the experimental values are in the predicted interval and the behavior of this parameter depends on the obtaining conditions of the samples. 

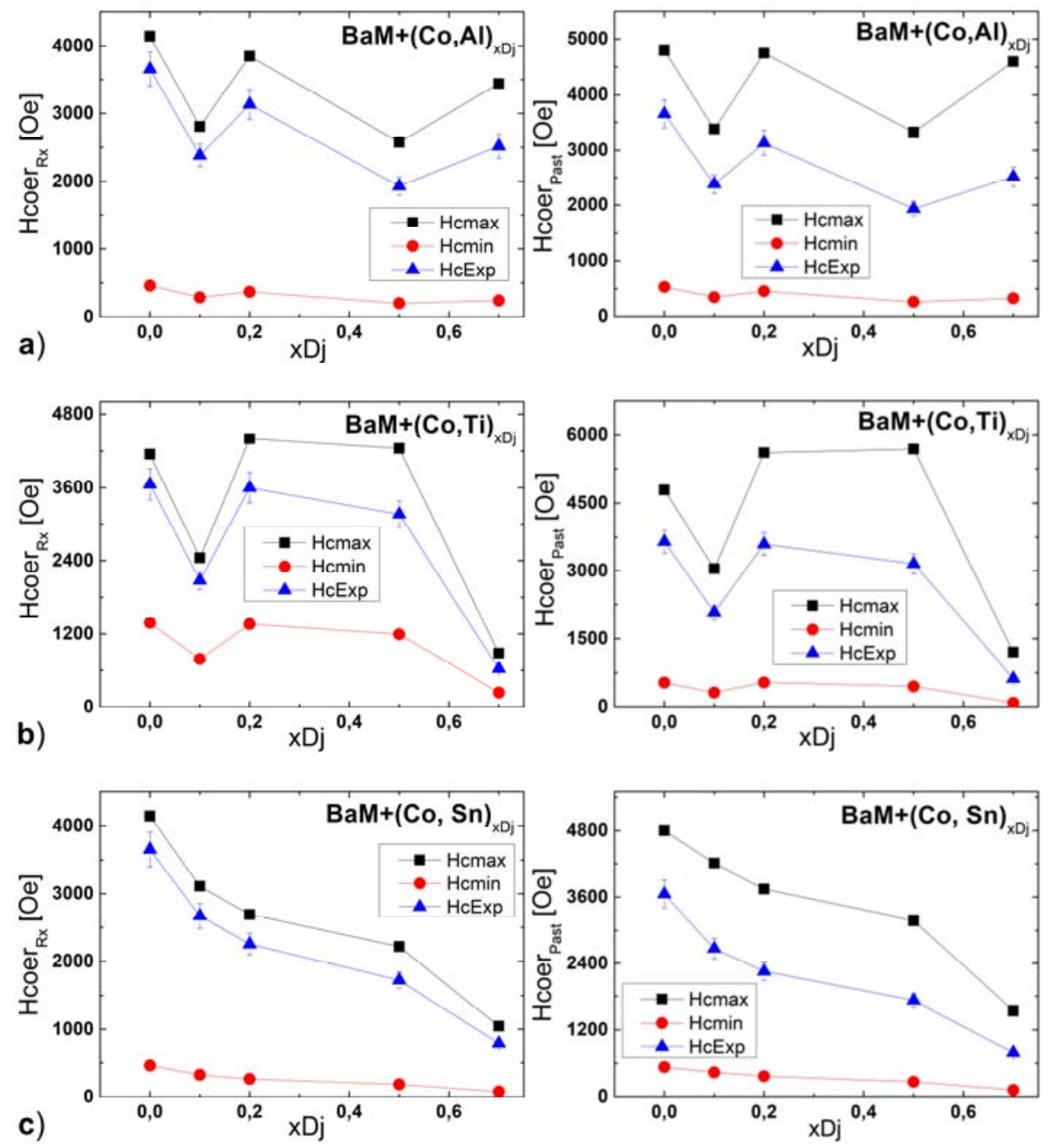

Figure 10. Comparison between theoretical and experimental coercive field $H_{C}$, considering the BaM theoretical density and the density of the pill, in function of the quantity of dopant per formula unit for the systems (a) BaM+(Co, $A l) x D j$, (b) BaM+(Co, Ti)xDj and (c) BaM+(Co, Sn)xDj.

For the substitutions with $(\mathrm{Co} 2+, \mathrm{Sn} 4+)$ there are bigger variations of the grain size and fractions of pores, while the variations of strain coefficient are small with $\mathrm{xDj} \leq 0.2$. All these facts condition the behavior in this region of the experimental curve. Starting from this value, the fraction of pores increases in a drastic way, decreasing the coercive field intensity.

\section{Conclusions}

The saturation magnetization depends on the contribution of each iron site to the total magnetization of the un-doped and doped samples with small dopant concentrations and on the obtaining conditions of the compound, which significantly influence in the formed phases.

Magneto-crystalline anisotropy constant depends on the contribution of each iron site and not only of the position $2 b$. Its magnitude is modulated by other factors that affect the experimental results and which are not considered by the model.

There were considerable variations between the systems, in dependence of substitutes type and their distribution in the iron sites. The comparison between the theoretical predictions and the experimental results has allowed a better approach to the physical interpretation of the causes that condition the variation of the magnetic properties with the type and the concentration of two substitutes ions inside the $\mathrm{BaM}$ cell.

\section{References}

[1] T. Kaur, B. Kaur, B. H. Bhat, S. Kumar and A. K. Srivastava, "Effect of calcination temperature on microstructure, dielectric, magnetic and optical properties of $\mathrm{Ba}_{0.7} \mathrm{La}_{0.3} \mathrm{Fe}_{11.7} \mathrm{Co}_{0.3} \mathrm{O}_{19}$ hexaferrites“, Physica B: Condensed Matter 456, (2015) 206-212.

[2] X. Zhang, Y. Zhang, Z. Yue, J. Zhang, "Influences of sintering atmosphere on the magnetic and electrical properties of barium hexaferrites", AIP Advances 9, 085129 (2019) 1-6.

[3] R. S. Alam, M. Moradi, M. Rostami, H. Nikmanesh, R. Moayedi, Y. Bai, J. Magn. Magn. Mater. 381 (2015) 1-9.

[4] P. A. Mariño, "Estudio de la hexaferrita de bario Tipo-M dopada: predicciones y resultados experimentales", 2012, La Habana Editorial Universitaria ISBN 978-959-16-19600 . 
[5] H. Nikmanesh, S. Hoghoghifard, B. Hadi-Sichani, "Study of the structural, magnetic, and microwave absorption properties of the simultaneous substitution of several cations in the barium hexaferrite structure", Journal of Alloys and Compounds 775 (2019) 1101-1108.

[6] P. A Mariño-Castellanos, A. C. Moreno-Borges, G. OrozcoMelgar, J. A. García and E. Govea-Alcaide, "Structural and magnetic study of the Ti4+-doped barium hexaferrite ceramic samples: Theoretical and experimental results", Physica B: Condensed Matter 406 (2011) 3130-3136.

[7] P. A. Mariño, V. A. Lapshinsky, C. Pupo, J. Matilla, and A. Vega, "Obtaining and Structural Characterization of M-type Hexaferrites Doped with Two Cations in the Fe 3+ Sites" in MEPhI's Section of the Scientific Session on "Breakthrough directions of scientific research at MEPhI: Development prospects within the Strategic Academic Units", KnE Engineering, (2018), pages 328-335.

[8] L. Glasser, "Lattice energies of crystals with multiple ions: a generalized Kapustinskii equation", 1995, Inorg. Chem. 34 4935-4936.

[9] J. Matilla-Arias, E. Govea-Alcaide, P. Mariño-Castellanos, F. Rosales-Saiz "Phenomenological Model for Prediction of
Cation Substitution Distribution and Some Physical Properties in $\mathrm{Mn}^{3+}$-Doped Barium Hexaferrite", J Supercond Nov Magn 31 (2018) 251-256.

[10] B. B. Rath, "Kinetics of nucleation and growth processes", July 1995, Materials Science and Engineering: B 32 (3) 101106.

[11] J. Matilla-Arias, E. Govea-Alcaide, P. Mariño-Castellanos, F. Rosales-Saiz, I. F. Machado, K. Montero-Rey, "Effects of Lanthanum on Structural and Magnetic Properties of $\mathrm{Sr}_{1-\mathrm{x}} \mathrm{La}_{2 \times / 3} \mathrm{Fe}_{12} \mathrm{O}_{19}$ Compounds: Theoretical and Experimental Results", JSupercondNovMagn (2019) 152-158.

[12] S. Asiri, S. Güner, A. D. Korkmaz, Md. Amir, K. M. Batoo, M. A. Almessiere, H. Gungunes, H. Sözeri, A. Baykal, " Magneto-optical properties of BaCryFe12-yO19 (0.0 $\mathrm{y} \leq 1.0)$ hexaferrites", Journal of Magnetism and Magnetic Materials 451 (2018) 463-472

[13] F. L Wei, H. C. Fang, C. K. Ong, C. S. Wang and Z. Yang, "Magnetic properties of $\mathrm{BaFe}_{12-2 \mathrm{x}} \mathrm{Zn}_{\mathrm{x}} \mathrm{Zr}_{\mathrm{x}} \mathrm{O}_{19}$ particles", June 2000, J. Appl. Phys. 87 (12) 8636-8639. 